\title{
MEMS Sensor for In Situ TEM Atomic Force Microscopy
}

\author{
Alexandra Nafari, David Karlen, Cristina Rusu, Krister Svensson, Håkan Olin, and Peter Enoksson
}

\begin{abstract}
Here, we present a MEMS atomic force microscope sensor for use inside a transmission electron microscope (TEM). This enables direct in situ TEM force measurements in the nanonewton range and thus mechanical characterization of nanosized structures. The main design challenges of the system and sensor are to reach a high sensitivity and to make a compact design that allows the sensor to be fitted in the narrow dimensions of the pole gap inside the TEM. In order to miniaturize the sensing device, an integrated detection with piezoresistive elements arranged in a full Wheatstone bridge was used. Fabrication of the sensor was done using standard micromachining techniques, such as ion implantation, oxide growth and deep reactive ion etch. We also present in situ TEM force measurements on nanotubes, which demonstrate the ability to measure spring constants of nanoscale systems.

[2007-0260]
\end{abstract}

Index Terms-Atomic force microscopy (AFM), force measurements, in situ transmission electron microscope (TEM), MEMS, nanowire characterization.

\section{INTRODUCTION}

$\mathbf{T}$ HE transmission electron microscope atomic force microscopy (TEM-AFM) is an important new member in a growing family of systems which are a combination of TEM and scanning probe microscopy (SPM) (for examples, see [1] and references therein). The TEM is used for localizing a suitable object to reveal the microstructure of both measurement probe and sample and to measure the probe-sample distance, probe and sample shape, etc. All this information is lacking and, thus, hampering standard SPM. The SPM implementation in the TEM-SPM provides the local probe and manipulator for in situ measurements; thus, properties not measured in standard TEM can be investigated. The main challenge when combining the SPM with TEM is the limited space available inside the TEM pole gap. This makes MEMS a good candidate for constructing the sensors or even the manipulators [2]. Earlier, direct in situ MEMS force sensors had been made for nanoindentation [3] or tensile testing [4] with force sensitivity in the micronewton

Manuscript received June 4, 2007; revised October 31, 2007. Subject Editor H. Zappe.

A. Nafari and D. Karlen are with the Department of Microtechnology and Nanoscience (MC2), Chalmers University of Technology, 41258 Gothenburg, Sweden, and also with the Nanofactory Instruments AB, 41296 Gothenburg, Sweden (e-mail: alexandra.nafari@chalmers.se).

C. Rusu is with the Imego Institute, 41133 Gothenburg, Sweden.

K. Svensson is with the Department of Physics, Karlstad University, 65188 Karlstad, Sweden.

H. Olin is with Mid Sweden University, 85170 Sundsvall, Sweden.

P. Enoksson is with the Department of Microtechnology and Nanoscience (MC2), Chalmers University of Technology, 41258 Gothenburg, Sweden.

Color versions of one or more of the figures in this paper are available online at http://ieeexplore.ieee.org.

Digital Object Identifier 10.1109/JMEMS.2007.912714

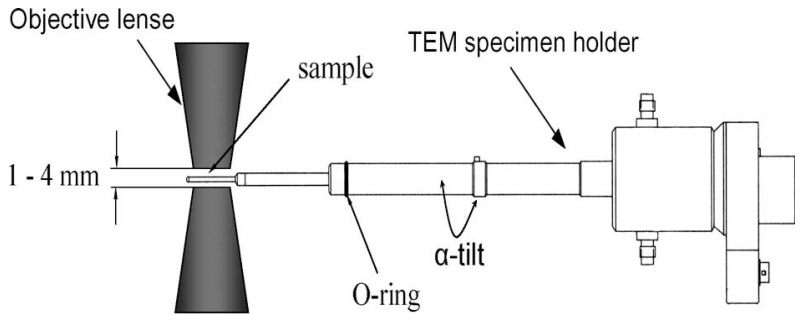

Fig. 1. TEM specimen holder between the upper and lower objective lens.

range. Previous attempts with forces in the nanonewton range are of two types. First, there is a very simple one using a standard AFM cantilever with a known spring constant which is placed inside the TEM [5], [6]. The deflection of the cantilever is then recorded using the TEM images, and the force can be calculated later using Hooke's law. The second type is an elaborate rebuilding of a TEM to incorporate a standard AFM instrument with optical detection [7].

Here, we report on the first MEMS AFM sensor with integrated detection for use inside a TEM. The TEM-AFM system has been evaluated by performing in situ force measurements on carbon nanotubes where the spring constant of a nanotube pair was measured. Such data in combination with structural dimensions measured in TEM can be used for extracting material properties such as Young's modulus.

\section{DESIGN}

The ultimate resolution of a TEM with a side-entry sample holder is highly dependent on the pole gap, i.e., the distance between the two objective lenses at both sides of the sample. This separation is typically $1-4 \mathrm{~mm}$ depending on the pole-piece design (usually, a smaller gap means a higher resolution). Fig. 1 shows a schematic picture of the specimen holder inserted in the pole gap. The specimen holders have a larger diameter further away from the pole gap, ensuring a high mechanical stability.

The specimen is placed in the front part of specimen holder, and the high vacuum in the TEM column extends up to the O-ring. It is desirable to rotate the specimen holder around its axis (the $\alpha$-direction), thus tilting the sample relative to the electron beam. This tilt will, however, restrict the dimensions of the TEM specimen holder further for a given pole gap. There are also other factors in the TEM environment that need to be considered when designing the system. The high vacuum in the TEM column will reduce the cooling of the device and increase the self-heating. There are also potential charging problems related to backscattered electrons and X-rays, which 


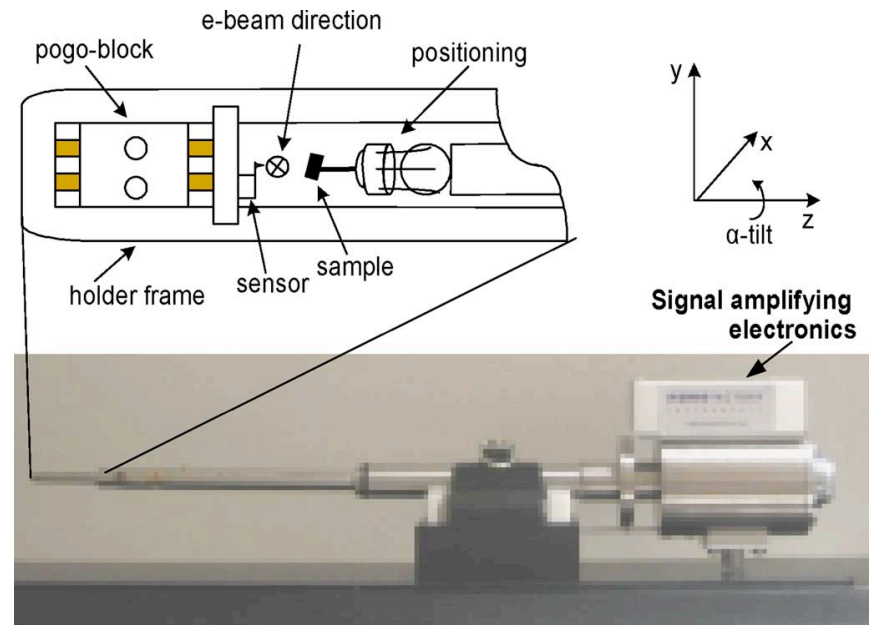

Fig. 2. Sideview image of the in situ probe holder and zoom in sketch showing the mounting of sample and sensor.

are generated as the electron beam hits the sample during imaging.

The first obvious challenge for an in situ TEM-AFM is to make the sensor small enough to be fitted in the restricted space of the pole gap. The holder front piece used here is 2.4-mm thick in order to fit inside a Phillips CM200 (Supertwin lens) without restricting the $\alpha$-tilt. The printed circuit board (PCB) on which the sensor is mounted has the dimensions of $4 \times 2.4 \mathrm{~mm}^{2}$ which, including margins for wire bonding, leaves $2.4 \times 1.3 \mathrm{~mm}^{2}$ for the base of the sensor. Another requirement is that the tip of the AFM cantilever preferably should be centered with respect to the electron beam, as shown in Fig. 2. This sets the final maximum sensor dimension to $1.2 \times 1.3 \times 0.5 \mathrm{~mm}^{3}$. Note that the sensor is mounted on the side when used in the holder (Fig. 2); therefore, the thickness of the sensor is not as critical as the width.

In order to miniaturize the sensing device, an integrated detection with piezoresistive elements arranged in a full Wheatstone bridge was chosen. For additional temperature compensation, one of the bridge resistors was placed on a dummy cantilever, as shown in Fig. 3(a). In order to decrease the influence of secondary electrons, the driving pads were designed to be closer to the electron beam. The electrons that are absorbed by the tip during imaging are drained through a substrate contact which is connected to one of the excitation pads. The TEM probe holder can be rotated about $30^{\circ}$ (in the $\alpha$-direction as shown in Figs. 1 and 2), and in order to avoid shadowing at high angles of rotation, the sensor front edge was chamfered to $20^{\circ}$ (see Fig. 3).

The manufactured sensor is glued and wire-bonded to a ceramic PCB, as shown in Fig. 3(b). The whole chip is then mounted in the TEM specimen holder. The ceramic PCB has via-hole connections to the backside where a pogo-block construction connects the electrical connection to the signal amplifying electronics on the holder shaft. The center hole in the PCB is an alignment mark used to center the cantilever tip during mounting.

The TEM probe holder used here is a modified TEM scanning-tunneling-microscopy single-tilt holder developed at Nanofactory Instruments [8]. The positioning system utilizes (a)
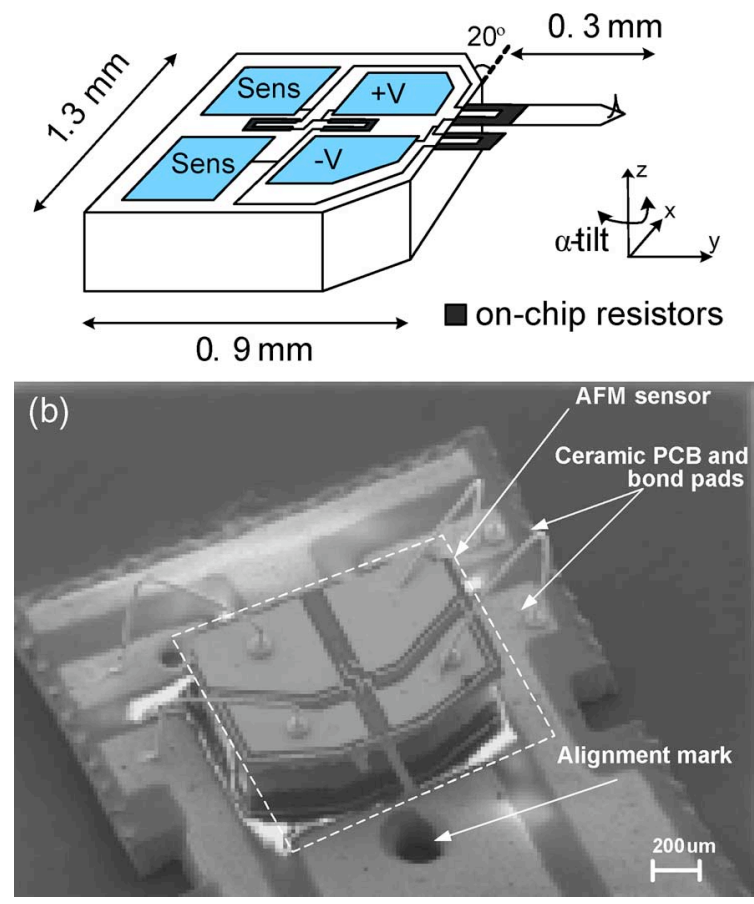

Fig. 3. (a) Sketch of the AFM sensor. (b) SEM image of the fabricated AFM sensor that is wire-bonded and mounted on a PCB.

piezoelectric movements and inertial-sliding coarse motions in three dimensions. This combines the long-range movement of the inertial sliding and the subnanometer accuracy of the piezotube [9]. The MEMS force sensor is placed on the nonmoving side to simplify electrical connections and to keep the sensor as mechanically stable as possible. The sample was glued onto a wire and placed on the moving part, as shown in Fig. 2.

\section{FABRICATION}

The AFM sensor was fabricated using silicon micromachining on an n-type SOI wafer. The major fabrication steps are schematically drawn in Fig. 4. The device layer thickness was $10 \mu \mathrm{m}$, and the resistivity was $1-10 \Omega \cdot \mathrm{cm}$. The first step in the process has two tasks: 1) to fabricate the tip and 2) to define the cantilever thickness. The cantilever thickness is obtained by thinning down the device layer by the same isotropic etch process used to fabricate the tip. In our case, the cantilever is about $3-\mu \mathrm{m}$ thick. To fabricate the tip, a thermally grown oxide was patterned and used as a mask material in an isotropic etch. The isotropic etch was done using $\mathrm{SF}_{6}$ plasma in an surface technology systems-inductively coupled plasma etch instrument. The tip is shaped by the under etch from the isotropic etch [see Fig. 4(b)]. For further sharpening, the wafers were thermally oxidized without removing the original oxide mask [see Fig. 4(c)]. Oxidation sharpening of silicon tips was first investigated by Ravi and Marcus [10]. This method is excellent for the fabrication of AFM tips with high yield over a $6^{\prime \prime}$ wafer. To protect the tip during further processing, the patterning is done using thick photoresist $(4-5 \mu \mathrm{m})$.

The $\mathrm{p}^{+}$-contacts to the resistors and the $\mathrm{n}^{+}$-contact to the device layer were diffusion-doped. The p-doping was done 


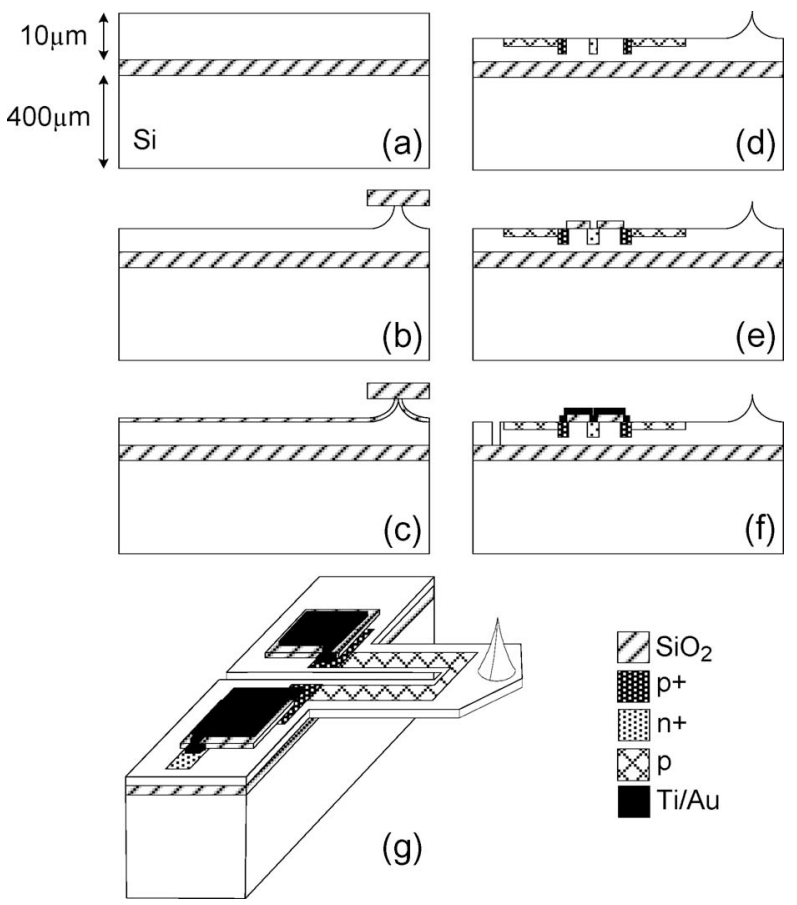

Fig. 4. Schematic view of the fabrication process. (a) SOI wafer used, device layer and handle wafer are 10- and $400-\mu \mathrm{m}$ thick, respectively, and the BOX is $2 \mu \mathrm{m}$. (b) The tip is etched using $\mathrm{SF}_{6}$ plasma. (c) Tip sharpening with thermal oxidation. (d) $\mathrm{p}^{+}-, \mathrm{n}^{+}$, and resistor doping is done. (e) Oxide is deposited for isolation of electrical pads. (f) Metal is deposited, and the cantilever is defined. (g) The cantilever is released with DRIE and buffered oxide etching. One out of four resistors is shown.

with boron, and the $\mathrm{n}$-doping was done with phosphorus. The $\mathrm{n}$-contact is made to have a connection to the bulk of the device layer. This connection has two aims: The first is to create a reverse-biased diode confining the current into the resistors, and the second is to drain the electrons that are absorbed by the tip during imaging with the e-beam. The resistors are formed by ion-implanting boron. The doses used are in the range of $1.5 \cdot 10^{14}-1.5 \cdot 10^{15} \mathrm{~cm}^{-2}$, with an implantation energy of $50 \mathrm{keV}$. The doses are chosen to give high piezoresistive coefficient and low temperature dependence [11]. To activate the doped areas, the wafers were annealed at $1000{ }^{\circ} \mathrm{C}$ for $1 \mathrm{~h}$. The relatively high temperature and long time were used to reduce noise induced by damages done to the crystalline structure during the implantation [12].

On-chip connections were made by depositing $\mathrm{Ti} / \mathrm{Au}$, as shown in Fig. 4(f). The metal was deposited and patterned on $1 \mu \mathrm{m}$ of plasma-enhanced chemical vapor deposition oxide [Fig. 4(e)] to avoid any direct contact between the silicon and the metal except for where the contact holes are. Furthermore, to ensure an ohmic contact, the wafers were annealed at $300{ }^{\circ} \mathrm{C}$ for $30 \mathrm{~min}$. To electrically isolate the resistors, the silicon around the resistors was etched down to the buried oxide (BOX) in the same step that the cantilever is defined. The final step is to release the cantilever and shape the base of the sensor. This etch is done using deep reactive ion etch (DRIE). With DRIE, it is more straightforward to fabricate small devices with arbitrary shapes than with wet etching.

During the last etch step where the whole wafer is etched through for releasing the cantilevers, the wafer becomes fragile and can easily break. To avoid this, the deep backside etch was carried out in two steps. First, the backside is etched down to $300 \mu \mathrm{m}$, and the wafer is diced into smaller units of $5 \times 5$ sensors. The etch is then continued on the diced units. The cantilever is finally fully released by etching the BOX in the SOI wafer [see Fig. 4(g)]. The BOX is etched using HF to ensure high selectivity and no damage to the tip.

The sensors are diced using a diamond saw. There are several issues to consider during dicing. First of all, mounting of the sensors is important. When the area of the sensor chip is on the order of a few square millimeters, a strong adhesive tape is needed to keep the sensor from loosening during dicing. Here, also the amount of cooling water should be kept at a minimum to avoid any extra forces on the sensor. The cantilevers are not damaged by the cooling water, but the sensors may loosen from the tape. The second important issue during dicing is the mechanical vibrations. The vibrations can break the cantilevers if they are in the range of the resonance frequency of the sensor. What dicing parameters to use in order to avoid this is dependent on the dicing tool used, and for our particular tool, the rotation speed was the parameter that affected the yield the most. A higher rotation speed than $20000 \mathrm{r} / \mathrm{min}$ resulted in a $50 \%$ yield, whereas at a lower rotation speed, the yield was nearly $100 \%$.

When the metal was deposited and patterned, the first electrical tests were performed. Measurements on test structures indicated an unwanted device layer substrate connection on the first batch of sensors. The stray connection was traced to a boron impurity close to the bonded interface in the SOI wafer in the device layer, i.e., the device layer is not uniformly n-type throughout the depth of the device layer as it should have been. Depending on the final cantilever thickness and heat treatments, the boron impurity and the resistors were not properly separated, which results in poor p-n junctions, thus inducing stray currents. A second batch of sensors was fabricated on SOI wafers from a different supplier. The electrical performance of the second batch was significantly better. This is still under investigations; however, first measurements using secondary ion mass spectrometry showed that the boron impurity was still present in the new SOI wafers, but the concentration was less.

The sensors from the first batch will, from here on, be referred to as versionA sensors and the second batch as versionB.

\section{Sensor Characterization}

The typical tip radius of the fabricated sensors was about $15 \mathrm{~nm}$, as shown from the TEM image in Fig. 5. The tip radius can be optimized further by better protection of the wafer during the process, for example, by changing the process so that the tip oxide can be kept longer. The sensors were characterized by pressing a sample onto the cantilever, thereby applying a force, and measuring response and noise. The force applied $(F)$ on the sensor can be obtained using Hooke's law

$$
\left\{\begin{array}{l}
F=k_{s} \cdot \Delta z \\
U=S \cdot \Delta z
\end{array} \Rightarrow F=\frac{k_{s} \cdot U}{S}\right.
$$




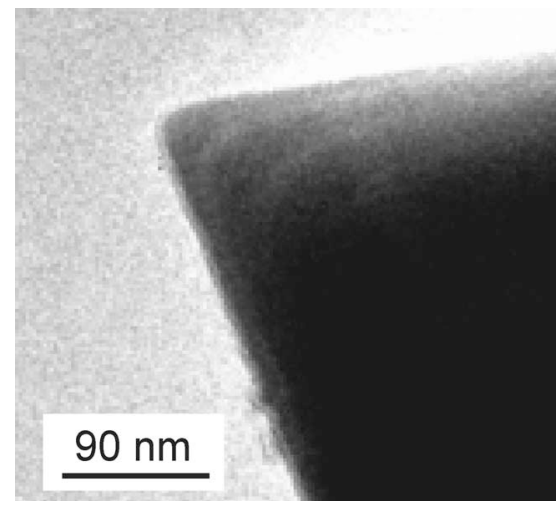

Fig. 5. TEM image of a manufactured AFM sensor tip. The tip radius is $15 \mathrm{~nm}$.

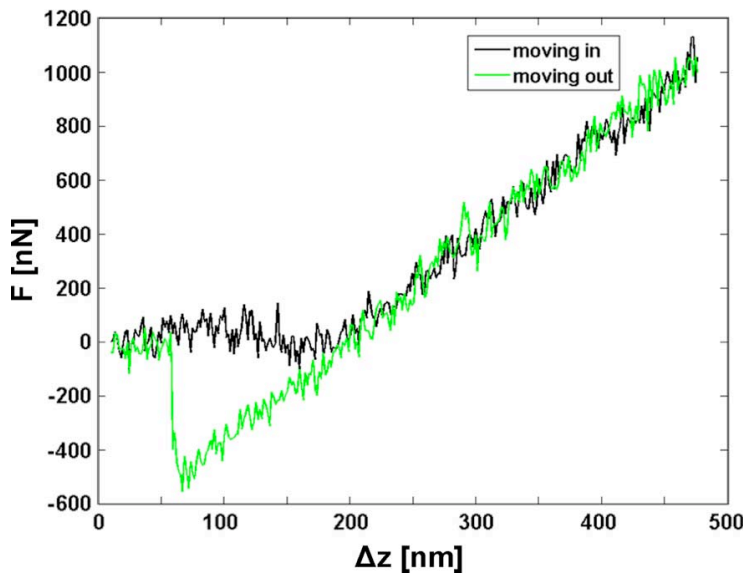

Fig. 6. Force measurement with versionA sensors on an aluminum surface used for calibration. The curve has been compensated for an offset of $200 \mathrm{nN}$ and a linear drift.

where $\mathrm{U}, \Delta z, \mathrm{~S}$, and $k_{s}$ are the bridge output, the distance that the cantilever is deflected, the sensor electrical sensitivity, and the spring constant of the sensor, respectively. $S$ is measured by pressing the cantilever against a hard surface while monitoring the displacement $\Delta z . k_{s}$ is calculated using the measured dimensions and simple beam-deflection theory.

The versionA sensors were characterized inside the TEM using an aluminum wire. Fig. 6 shows a measured force-displacement curve. During retraction, the common hysteresis in the force curve is observed. This is usually due to surface forces, but the high magnitude of the force observed here suggests that additional adhesion forces were present such as welding caused by a high e-beam exposure in combination with poor vacuum. The peak-to-peak TEM-AFM system noise when operating in the TEM was measured to $100 \mathrm{nN}$, corresponding to $30 \mu \mathrm{V}$. The noise level was the same for measurements in air. The shielding from a $50-\mathrm{Hz}$ noise induced by surrounding electrical environment was, however, better in the TEM, presumably due to shielding by the TEM column.

In order to analyze the frequency distribution of the noise, measurements were done using the TEM-AFM holder (Fig. 2) and electronics on both versionA and versionB sensors and compared to a reference bridge setup. The reference Wheat-

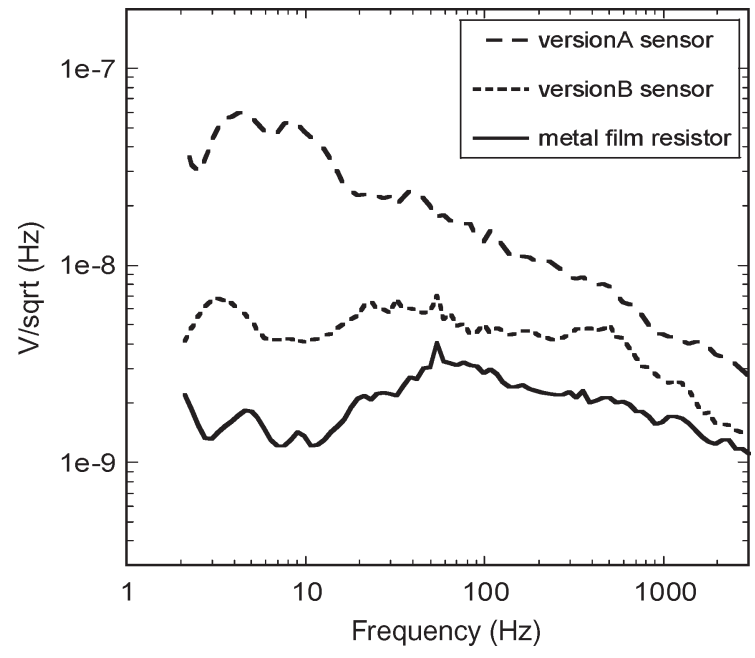

Fig. 7. Noise spectrum of versionA, versionB, and metal-film resistors. The curves have been smoothed to better show the trends.

stone bridge consisted of metal-film resistors mounted on a PCB that would fit inside the holder in order to have the same configuration in all three setups. The obtained signals were Fourier-transformed using Matlab, and the results are presented in Fig. 7.

As shown in Fig. 7, the $1 / f$ noise in versionA sensors is dominating, and the amplitude of it is one magnitude higher than for versionB sensors at low frequencies. As mentioned earlier, we believe that this noise originates from a high boron concentration close to the BOX in the n-type SOI wafer used. In versionB sensors, where this problem had been solved, the noise follows the reference bridge's noise curve with an offset. This indicates that the noise level of the versionB sensors is close to the electronic noise. The noise density of the versionB sensor is at an acceptable limit for low-frequency applications such as scanning.

The versionB sensors were further evaluated in air on a gold sample. From the data in the force plot in Fig. 8(a), the peakto-peak noise in the measurement can be estimated to $15 \mathrm{nN}$, corresponding to $6.5 \mu \mathrm{V}$. The expected snap-in and snap-out are clearly seen, as the sample is moved in toward the sensor and retracted. The versionB sensors were also used for scanning a chessboardlike grating made of silicon with arrays of square pillars, as shown in Fig. 8(b).

\section{In Situ TEM Measurements}

The TEM-AFM system versatility was tested using ironfilled carbon nanotubes. The nanotubes were pressed into the cantilever tip while imaging (using the TEM) and monitoring the force in real time. Fig. 9 shows a TEM image of the nanotube with the corresponding force-distance plot. VersionA sensors were used for this experiment, as the versionB sensors were not available at the time. The spring constant of the cantilever was calculated from the measured dimensions to $2.9 \mathrm{~N} / \mathrm{m}$. When comparing the AFM tip shown in Figs. 5 and 9 (a), it is seen that the tip radius differs. This is primarily due to the decomposition of carbon inside the TEM. This deposition 


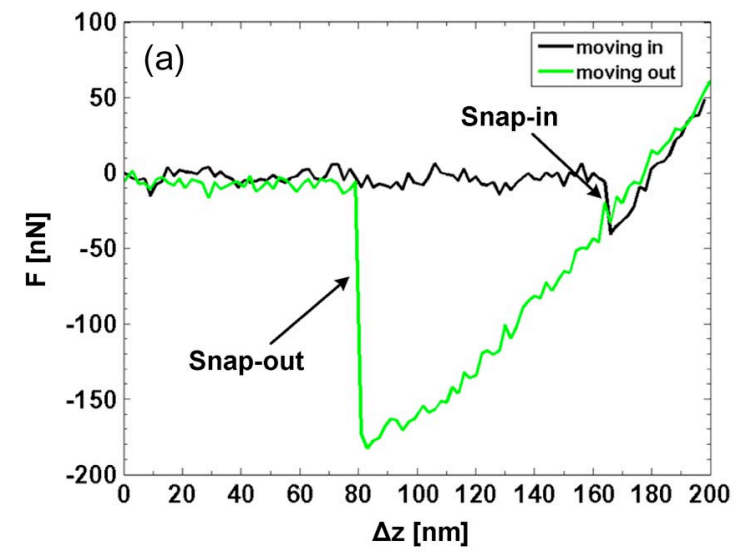

(b)

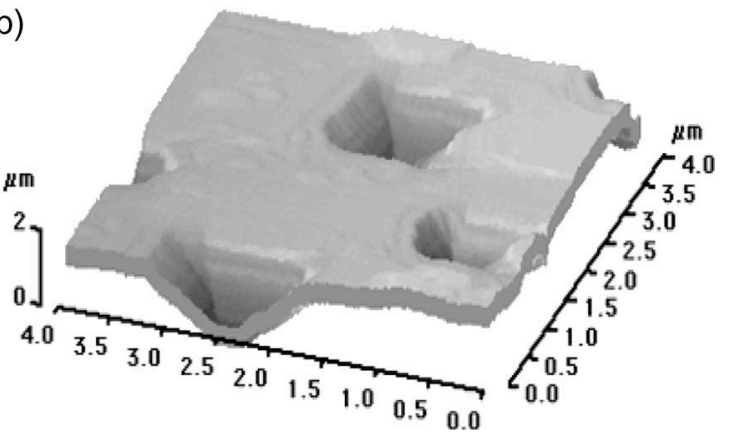

Fig. 8. Measurements in air with versionB sensors. (a) Force-displacement curve on a gold sample. (b) A scan of a chessboard grating.

of amorphous carbon inside the TEM blunts the AFM tip with time and could possibly be reduced by using plasma cleaner to clean the sample area of the holder before insertion into the TEM.

During the in situ TEM measurement, a drift in the offset level of the sensor output was observed. As the electron beam hits the sample and the cantilever, secondary electrons and $\mathrm{X}$-rays are emitted. The measurement signal can drift due to a charge-induced current. The offset level drifted typically during the alignment of the TEM beam when the magnification was low and thicker parts of sample and cantilever were illuminated. There, the electrons are not transmitted through the sample, and a large amount of secondary electrons and X-rays are generated. To decrease drift, heavier metals, such as gold, should be avoided as a sample support as they emit more secondary electrons and X-rays. The drift in offset is also considerably reduced when working at high magnifications, and only the electron transparent regions of the sample and tip are illuminated. From the force plot, it is possible to extract the force, the displacement, and the spring constant of the complete system $\left(k_{\text {tot }}\right)$ which are related according to Hooke's law, $\Delta z=F / k_{\text {tot }}$. However, the force situation is somewhat more complicated than that. To extract the spring constant of the nanoscale system of interest, we have to consider that the cantilever is also flexible. The total spring constant $k_{\text {tot }}$ can be expressed in terms of the spring constants of the silicon cantilever and the nanotubes as

$$
k_{\mathrm{tot}}=\frac{k_{s} k_{\mathrm{nt}}}{k_{s}+k_{\mathrm{nt}}}
$$
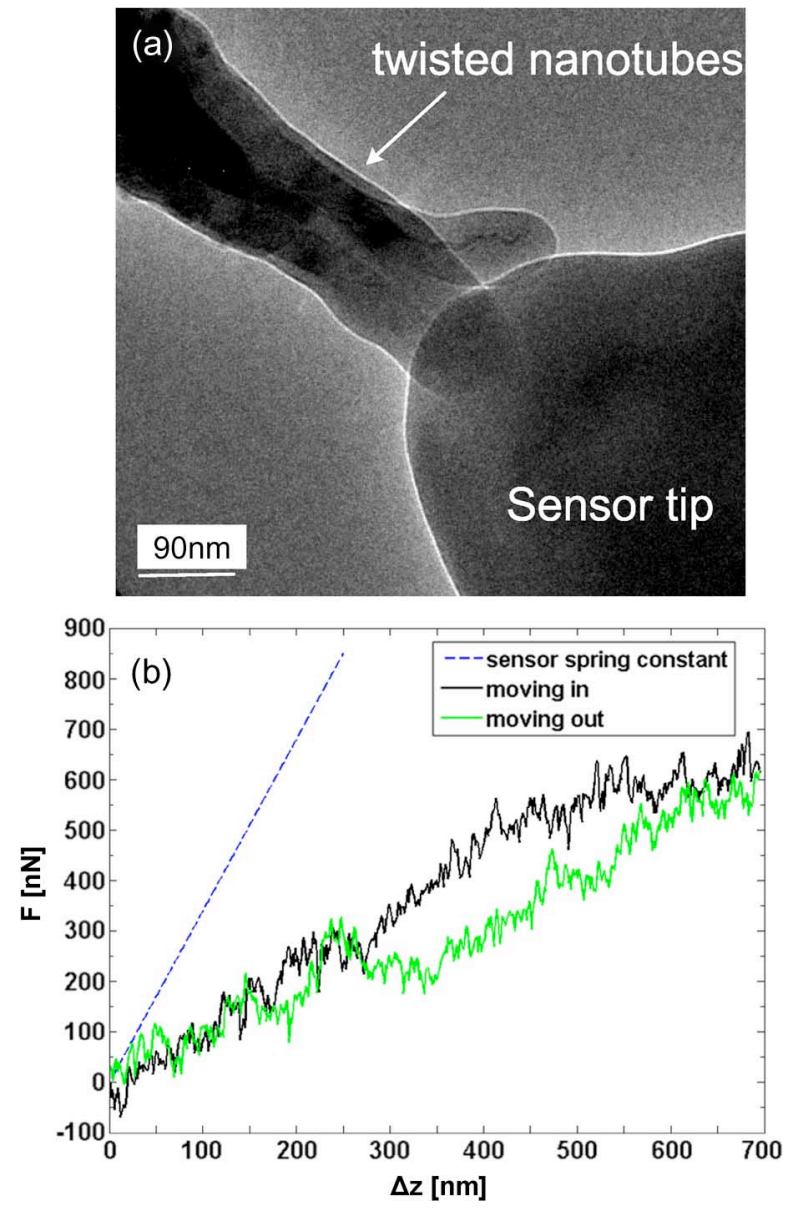

Fig. 9. In situ TEM measurements on nanotubes using versionA sensor. (a) TEM image of the iron-filled nanotubes. (b) Corresponding forcedisplacement plot.

where $k_{s}$ and $k_{\mathrm{nt}}$ are the spring constants of the sensor and nanotubes, respectively. The total spring constant is estimated from the force-distance plot in Fig. 9(b) to be $1 \mathrm{~N} / \mathrm{m}$, giving a spring constant of the twisted nanotubes of $1.5 \mathrm{~N} / \mathrm{m}$.

These measurements demonstrate the ability of the system to measure spring constants of nanoscale systems. To be able to extract detailed material properties from the measurements, e.g., Young's modulus, the dimensions and geometries need to be carefully measured.

\section{CONCLUSION}

An in situ TEM-AFM system utilizing a compact MEMS sensor has been developed and evaluated. The MEMS sensor has the dimensions of $1.2 \times 1.3 \times 0.5 \mathrm{~mm}^{3}$ and is small enough to be integrated in a modified standard TEM holder with no changes to the outer dimensions. The TEM-AFM system can therefore be used without any alterations to the TEM. The MEMS force sensor is fabricated using standard micromachining methods. The sensor has a full Wheatstone bridge integrated on chip enabling a compact design and high sensitivity. The system has also been used for scanning a surface.

The versatility of sensor and system was verified by obtaining the spring constant for a nanoscale system inside a TEM. 


\section{REFERENCES}

[1] K. Svensson, H. Olin, and E. Olsson, "Nanopipettes for metal transport," Phys. Rev. Lett., vol. 93, no. 14, p. 14 590, Oct. 2004.

[2] M. I. Lutwyche and Y. Wada, "Manufacture of micromechanical scanning tunnelling microscopes for observation of the tip apex in a transmission electron microscope," Sens. Actuators A, Phys., vol. 48, no. 2, pp. 127136, May 1995.

[3] A. Nafari, H. A.Danilov, P. Rodjegard, and H. Enoksson, "A micromachined nanoindentation force sensor," Sens. Actuators A, Phys., vol. 123, pp. 44-49, Sep. 2005.

[4] M. A. Haque and M. T. A. Saif, "Application of MEMS force sensors for in situ mechanical characterization of nano-scale thin films in SEM and TEM,”Sens. Actuators A, Phys., vol. 97/98, pp. 239-245, Apr. 2002.

[5] D. Erts, A. Lõhmus, R. Lõhmus, H. Olin, A. V. Pokropivny, L. Ryen, and K. Svensson, "Force interactions and adhesion of gold contacts using a combined atomic force microscope and transmission electron microscope," Appl. Surf. Sci., vol. 188, no. 3/4, pp. 460-466, Mar. 2002.

[6] T. Kuzumaki and Y. Mitsuda, "Nanoscale mechanics of carbon nanotube evaluated by nanoprobe manipulation in transmission electron microscope," Jpn. J. Appl. Phys., vol. 45, no. 1A, pp. 364-368, 2006.

[7] T. Kizuka, H. Ohmi, T. Sumi, K. Kumazawa, S. Deguchi, M. Naruse, S. Fujisawa, S. Sasaki, A. Yabe, and Y. Enomoto, "Simultaneous observation of millisecond dynamics in atomistic structure, force, and conductance on the basis of transmission electron microscopy," Jpn. J. Appl. Phys. 2, Lett., vol. 40, no. 2B, pp. L170-L173, 2001.

[8] Nanofactory Instruments. [Online]. Available: www.nanofactory.com

[9] K. Svensson, Y. Jomopol, H. Olin, and E. Olsson, "Compact design of a transmission electron microscope-scanning tunneling microscope with three-dimensional coarse motion," Rev. Sci. Instrum., vol. 74, no. 11, p. 4945, Nov. 2003.

[10] T. S. Ravi and R. B. Marcus, "Oxidation sharpening of silicon tips," J. Vac. Sci. Technol., vol. 9, no. 6, pp. 2733-2737, Nov. 1991.

[11] Y. Kanda, "A graphical representation of the piezoresistance coefficients in silicon," IEEE Trans. Electron Devices, vol. ED-29, no. 1, pp. 64-70, Jan. 1982.

[12] L. K. J. Vandamme and S. Oosterhoff, "Annealing of ion-implanted resistors reduces the 1/f noise," J. Appl. Phys, vol. 59, no. 9, pp. 3169-3173, May 1986.

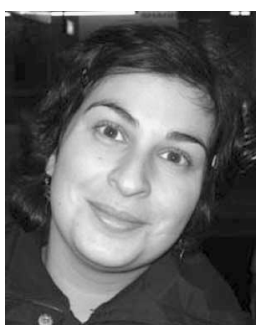

Alexandra Nafari received the M.S. and Lic. Eng. degrees in electrical engineering from the Chalmers University of Technology, Gothenburg, Sweden, in 2004 and 2007, respectively. She is currently working toward the Ph.D. degree in the Department of Microtechnology and Nanoscience (MC2), Chalmers Chalmers University of Technology, and Nanofactory Instruments $\mathrm{AB}$, Gothenburg.

Her main research activities are in the field of microsystems technology and the integration of it with in situ transmission electron microscopy manipulation and measurements.

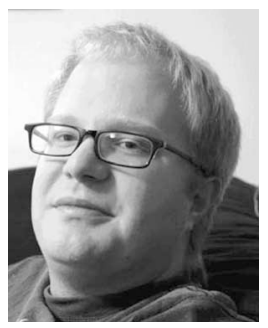

David Karlen received the M.Sc. degree in engineering physics and the Licentiate degree from the Chalmers University of Technology, Gothenburg, Sweden, in 2004 and 2007, respectively.

$\mathrm{He}$ is currently with the Department of Microtechnology and Nanoscience (MC2), Chalmers University of Technology, and also with Nanofactory Instruments $\mathrm{AB}$, Gothenburg. His research is focused on MEMS devices such as micro XY-stage and AFM sensors.

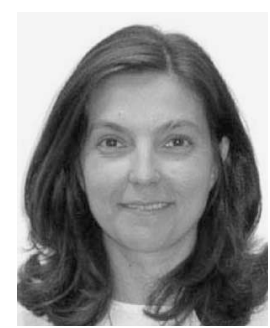

Cristina Rusu received the M.Sc. degree in applied physics from the University of Bucharest, Bucharest, Romania, in 1990 and the Ph.D. degree, with focus on micromachining techniques for biomedical application, from the University of Twente, Enschede, The Netherlands, in 1998.

From 1990 to 1994, she was with the Institute of Atomic Physics, Bucharest. From 1998 to 2000, she had postdoctoral fellowship in the Biophysical Technique Group, University of Twente. In 2000, she joined IMEC, Leuven, Belgium, to develop MEMS techniques for thin-film (poly-SiGe) encapsulation on the wafer level. Since August 2002, she has been with the Imego Institute, Gothenburg, Sweden, as a Senior Scientist, focusing on (wireless) microsensors and MEMS packaging technologies. In 2006, she received the title of Associate Professor from the Chalmers University of Technology, Gothenburg.

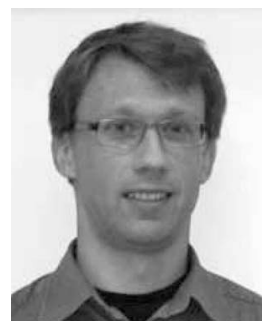

Krister Svensson received the B.Sc. degree in physics from Gothenburg University, Gothenburg, Sweden, in 1992 and the Licentiate and Ph.D. degrees in physics from the Chalmers University of Technology, Gothenburg, in 1995 and 1997, respectively.

He received a Marie Curie training and mobility grant and was a Postdoc at the University of Birmingham, Birmingham, U.K. until 2000. He then returned to the Chalmers University of Technology as an Assistant Professor and became a Researcher in 2004. Since 2007, he has been an Associate Professor in the Department of Physics, Karlstad University, Karlstad, Sweden. His main research interests lie within in situ TEM probing and the characterization of nanoscale structures such as carbon nanotubes. He is a co-holder of several patents involving the incorporation of scanning probe techniques into transmission electron microscopes, of which several have also been commercialized.

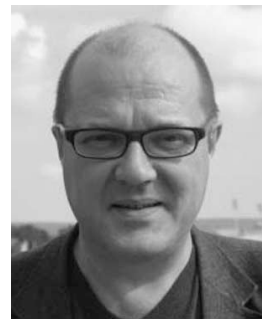

Håkan Olin received the Ph.D. degree from the Chalmers University of Technology, Gothenburg, Sweden, in 1993.

He was an Associate and Assistant Professor in solid-state physics at the Chalmers University of Technology. Since 2003, he has held the Chair in experimental material physics at Mid Sweden University, Sundsvall, Sweden. His research interest includes the fabrication and properties of nanostructures. The main methods have been scanning probe microscopy and nanofabrication, with applications in a wide area, including surfaces, nanowires, and nanodevices. In particular, he has done pioneering work on the in situ TEM probing technique.

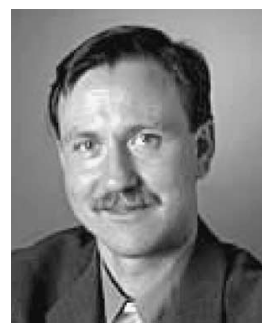

Peter Enoksson received the M.Sc. degree in engineering physics and the Licentiate of Engineering and Ph.D. degrees from the Royal Institute of Technology, Stockholm, Sweden, in 1986, 1995, and 1997, respectively.

He became an Assistant Professor in December 1997 and was appointed as an Associate Professor in 2000 at the Royal Institute of Technology, where he was a Supervisor and Project Leader in the Microsystem Technology Group. He has also taken a very active part in developing and giving courses in microsystems and measurement systems technology. In 2001, he was appointed as a Professor in the Department of Microtechnology and Nanoscience (MC2), Chalmers University of Technology, Gothenburg, Sweden, where he was appointed as the Vice Dean of the School of Electrical Engineering in 2002 and as the Head of the Solid State Electronics Laboratory in 2003. His main research activities are in combining MEMS/NEMS with other sciences in building more dedicated and advanced systems. He has published more than 100 research journal and conference papers. He is a referee for several journals in the field of MEMS. He is the holder of two patents, with three more pending. 